\title{
A necessary stabilization condition for PID control
}

\author{
Naim Bajcinca
}

\begin{abstract}
A necessary condition for the existence of a stable PID controller for a given plant is introduced. The derived rule is straightforward to check and applies generally for LTI systems, including time-continuous, time-discrete and timedelay systems. Therefore the plot of a directly constructible function is checked for a required minimal number of real zeros.
\end{abstract}

\section{INTRODUCTION}

PID controllers count to the simplest and most applied controller structures in a wide spectrum of industrial applications. Yet challenging theoretical problems have been recently formulated in searching for alternative procedures for the design of robust PID controllers to the standard tuning rules. A typical such problem is computation of the set of all robust PID stabilizers for a given finite set or a continuum of plants. The competition with the tuning rules approach requires simple solutions for non-trivial problems.

This paper presents a new necessary condition for the stabilizability of a time-continuous or a time-discrete PID loop, which additionally may include time delay. The rule is based on the decoupling of the PID parameter space $\left(k_{P}, k_{I}, k_{D}\right)$ at singular frequencies. Such conditions enable the construction of an equation of the form $k_{P}=k_{P}(\omega)$, which plays the central role in this work. Then, it is shown that for Hurwitz-stabilizability a $k_{P}$ must exist, such that the equation $k_{P}=k_{P}(\omega)$ possesses at least a fixed number of real-roots (singular frequencies) in the interval $(0,+\infty)$. This condition may be directly checked by plotting the function $k_{P}=k_{P}(\omega)$. The rule is then extended for Hurwitz-stability of time-delay systems and Schur-stability of time-discrete PID controllers. In the latter case, the notion of the decoupling function over the unity circle is introduced. Eventually, the usability of the rule is illustrated by several examples.

\section{DeCOUPLing}

\section{A. Time-continuous systems}

Consider Hurwitz-stability of the characteristic polynomial of a feedback-loop with a PID controller

$$
p(s)=A(s)\left(k_{I}+k_{P} s+k_{D} s^{2}\right)+B(s)
$$

where $A(s)$ and $B(s)$ are given real polynomials. No restriction w.r.t. the order of the polynomials $A$ and $B$ is made in this paper. The characteristic equation of (1) for

\footnotetext{
N. Bajcinca is with Institute of Robotics and Mechatronics at DLR, German Aerospace Center, Münchnerstr. 21, 82234, Oberpfaffenhofen, Germany; email: naim.bajcinca@dlr.de.
}

Hurwitz stability, $s=j \omega$, may be represented in the matrix form as follows

$$
\left[\begin{array}{l}
H \\
G
\end{array}\right]=\mathcal{P}_{A}\left[\begin{array}{cc}
1 & -\omega^{2} \\
0 & 0
\end{array}\right]\left[\begin{array}{c}
k_{I} \\
k_{D}
\end{array}\right]+\mathcal{P}_{A}\left[\begin{array}{c}
0 \\
\omega k_{P}
\end{array}\right]+\mathcal{P}_{B}=0
$$

where

$$
\mathcal{P}_{A}=\left[\begin{array}{cc}
R_{A} & -I_{A} \\
I_{A} & R_{A}
\end{array}\right], \mathcal{P}_{B}=\left[\begin{array}{c}
R_{B} \\
I_{B}
\end{array}\right]
$$

and $R$ and $I$ stand for real and imaginary part of polynomials $A$ and $B$ at $s=j \omega$. Notice that for a fixed $k_{P}$ in (2) the system matrix in terms of parameters $k_{I}$ and $k_{D}$ is $\forall \omega$ singular

$$
\operatorname{det} \frac{\partial(H, G)}{\partial\left(k_{I}, k_{D}\right)}=0, \quad \forall \omega .
$$

Yet (2) may have solutions at a finite set of frequencies $\left\{\omega_{1}^{\prime}, \omega_{2}^{\prime}, \cdots, \omega_{N}^{\prime}\right\}$, which are usually called isolated or singular frequencies. By left multiplication of (2) with $\mathcal{P}_{A}^{-1}$, the following two equations result

$$
\begin{aligned}
k_{I}-\omega^{2} k_{D}-\frac{R_{A} R_{B}+I_{A} I_{B}}{R_{A}^{2}+I_{A}^{2}} & =0 \\
\omega k_{P}-\frac{R_{A} I_{B}-I_{A} R_{B}}{R_{A}^{2}+I_{A}^{2}} & =0 .
\end{aligned}
$$

The importance of the latter representation is that the parameters of the PID controllers appear decoupled into two separate equations. In this paper, special attention is paid to the second equation (5), which has been referred to as the generator of singular frequencies or generator of isolated frequencies. Namely, for a fixed value of $k_{P}$ its real solutions $\left\{\omega_{1}^{\prime}, \omega_{2}^{\prime}, \cdots, \omega_{N}^{\prime}\right\}$ represent singular frequencies. The term 'singular' or 'isolated' addresses the fact that for a fixed $k_{P}$ the eigenvalues can cross the imaginary axis only at these frequencies. Furthermore, equation (4) maps each isolated frequency to a straight line in the $\left(k_{I}, k_{D}\right)$-plane, which, in turn, compose convex stable polygonal regions, [1]. Eventually, here it is important to mark that the generator of singular frequencies represents the imaginary part of the function

$$
F(s)=\frac{p(s)}{A(s)}=k_{I}+k_{P} s+k_{D} s^{2}+\frac{B(s)}{A(s)} .
$$

\section{B. Time-delay systems}

Hurwitz-stability of a PID loop with a time-delay $T_{d}$ is described by a quasi-polynomial of the form

$$
p(s)=A(s)\left(k_{I}+k_{P} s+k_{D} s^{2}\right)+B(s) e^{T_{d} s} .
$$


Following similar steps as in the previous subsections it can be easily shown that the quasi-polynomial (7) decouples also at isolated frequencies into the following two equations

$$
\begin{array}{r}
k_{I}-\omega^{2} k_{D}-\alpha(\omega) \cos \left(\omega L_{d}+\phi(\omega)\right)=0 \\
\omega k_{P}-\alpha(\omega) \sin \left(\omega L_{d}+\phi(\omega)\right)=0
\end{array}
$$

with

$$
\alpha(\omega)=\sqrt{\frac{R_{B}^{2}+I_{B}^{2}}{R_{A}{ }^{2}+I_{A}{ }^{2}}}, \quad \tan \phi(\omega)=\frac{R_{A} I_{B}-I_{A} R_{B}}{R_{A} R_{B}+I_{A} I_{B}} .
$$

Again, equation (9) represents the generator of singular frequencies. However, for a fixed $k_{P}$ infinite singular frequencies result now.

\section{Time-discrete systems}

To show that decoupling of PID controller applies also in the discrete time case, some definitions and pre-discussion is required. Though the derived results apply directly or are easily generalized for every simple and closed $\Gamma$ region in the $z$-plane, our main concern here is the Schur-stability and the unity circle

$$
\Gamma=\left\{e^{j \alpha}: \alpha \in[-\pi, \pi]\right\} .
$$

First, note that the characteristic equation of a discretetime PID control loop is of the form

$$
p(z)=A(z) Q(z)+B(z)=0
$$

where

$$
Q(z)=k_{I} z^{2}+k_{P} z(z-1)+k_{D}(z-1)^{2} .
$$

It is easy to check that the condition (3) does not apply $\forall \alpha$ on the unity-circle $\Gamma$, that is (12) is impossible to decouple over $\Gamma$. However, a $Q$ of the form

$$
Q(z)=\delta_{1}(z) r_{1}+\delta_{2}(z) r_{2}+r_{3}
$$

with

$$
\delta_{1}(z)=1+z^{2}, \quad \delta_{2}(z)=z
$$

satisfies this condition with respect to parameters $r_{1}$ and $r_{2}$, i.e.

$$
\operatorname{det} \frac{\partial(H, G)}{\partial\left(r_{1}, r_{2}\right)}=0, \quad \forall \alpha .
$$

For the equations (12) and (13) to be identical an appropriate liear parameter transformation $\left(k_{I}, k_{P}, k_{D}\right)^{T}=$ $T\left(r_{1}, r_{2}, r_{3}\right)^{T}$ can be easily solved for. Clearly the above expressions for $\delta_{1}(z)$ and $\delta_{2}(z)$ are not unique.

Definition 1: A function $E_{\Gamma}(z)$ defined as

$$
Q(z)=E_{\Gamma}(z) q(z)
$$

such that

$$
I_{q}=r_{3} g_{1}(\alpha)+g_{0}(\alpha), \quad \forall \alpha
$$

i.e. $\frac{\partial I_{q}}{\partial r_{1}}=\frac{\partial I_{q}}{\partial r_{2}}=0$, where $I_{q}$ stands for the imaginary part of $q$, will be referred to as the decoupling function of $Q$ over $\Gamma$.
Lemma 1: If (14) applies everywhere on $\Gamma$ then

$$
\frac{\partial I_{q}}{\partial r_{1}}=0 \Leftrightarrow \frac{\partial I_{q}}{\partial r_{2}}=0, \quad \forall z \in \Gamma .
$$

Proof. The reader can easily check that

$$
\begin{aligned}
\operatorname{det} \frac{\partial(H, G)}{\partial\left(r_{1}, r_{2}\right)}= & \left(R_{A}^{2}+I_{A}^{2}\right)\left(R_{E}^{2}+I_{E}^{2}\right) \\
& \times\left(\frac{\partial R_{q}}{\partial r_{1}} \frac{\partial I_{q}}{\partial r_{2}}-\frac{\partial I_{q}}{\partial r_{1}} \frac{\partial R_{q}}{\partial r_{2}}\right)
\end{aligned}
$$

If (14) applies, that is, the right-hand side of the above equation is zero, then $\frac{\partial I_{q}}{\partial r_{1}}=0 \Rightarrow \frac{\partial I_{q}}{\partial r_{2}}=0$, since $\frac{\partial R_{q}}{\partial r_{1}} \neq 0$. Similarly, $\frac{\partial I_{q}}{\partial r_{2}}=0 \Rightarrow \frac{\partial I_{q}}{\partial r_{1}}=0$.

Theorem I: $\delta_{1}(z)$ and $\delta_{2}(z)$ in (13) decouple $Q$ over $\Gamma$.

Proof. Suppose $E_{\Gamma}(z)=\delta_{1}(z)$. Then

$$
q=r_{1}+\frac{\delta_{2}(z)}{\delta_{1}(z)} r_{2}+\frac{1}{\delta_{1}(z)} r_{3},
$$

and $\frac{\partial I_{q}}{\partial r_{1}} \equiv 0$. Lema 1 guarantees that for all $z \in \Gamma$ the imaginary part of $\frac{\delta_{2}(z)}{\delta_{1}(z)}$ is also zero, that is, (16) applies. Since $\frac{\delta_{2}(z)}{\delta_{1}(z)}$ is real on $\Gamma$, its inverse will be also real, that is, the imaginary part of $\frac{\delta_{1}(z)}{\delta_{2}(z)}$ for all $z \in \Gamma$ is zero. Hence, $E_{\Gamma}=\delta_{2}(z)$ represents also a decoupling function.

The next theorem may be now directly stated.

Theorem 2: Consider the function

$$
F(z):=\frac{p(z)}{A(z) E_{\Gamma}(z)} .
$$

The equation $F(z)=0$ for $z \in \Gamma$ decouples the parameters $r_{1}, r_{2}$ and $r_{3}$ into two equations,

$$
\begin{aligned}
r_{1} h_{1}(\alpha)+r_{2} h_{2}(\alpha)+h_{0}(\alpha) & =0 \\
r_{3} g_{1}(\alpha)+g_{0}(\alpha) & =0 .
\end{aligned}
$$

Note that the second equation (20) is the sought generator of singular frequencies for the time-discrete PID controllers.

\section{THE MAIN RESULTS}

Based on the principle of argument simple necessary conditions for the stability of a PID loop are derived in this section. The results presented in this section use the decoupling of PID parameter space, shown in the previous section. For a more concise formulation of the Hurwitzstabilizability conditions an even function $E: \mathbb{N} \mapsto \mathbb{N}^{+}$ is defined to map a positive natural number to the nearest smaller even number.

\section{A. Time-continuous PID}

To prove the main result of this subsection the following lemma is needed.

Lemma 2: Consider the Mikhailov plot of a real-rational function $F(s)$ for $s=j \omega$ with $0 \leq \omega<+\infty$, and let $F(\infty) \rightarrow \infty$. If the net phase change of the Mikhailov 
vector $F(j \omega)$ is $N \pi / 2$, and $F(s)$ has no poles on the imaginary axis, then it cuts the real axis $Z$-times, where

$$
Z \geq \frac{1}{2} E(N)+1
$$

If $F(s)$ has $L$ poles on the imaginary axis, which are left circumscribed, then

(a) for $F(0) \neq \infty$

$$
Z \geq \frac{1}{2} E(N-L)+1
$$

(b) for $F(0)=\infty$

$$
Z \geq \frac{1}{2} E(N-L)
$$

A direct consequence reads as follows.

Lemma 3: If the net phase change of the Mikhailov vector $F(j \omega)$ for $0 \leq \omega<+\infty$ is $N \pi / 2$, and $F(s)$ has $L$ poles on the imaginary axis, which are all left circumscribed, then $F(j \omega)$ will intersect the real axis at $Z$ nonzero frequencies, where

$$
Z \geq \frac{1}{2} E(N-L)
$$

Now we are ready to introduce one of the key results of the paper.

Theorem 3: Consider the characteristic polynomial (1). Assume that the polynomial $A(s)$ has no zeros on the imaginary axis $j \omega$ and let

$N$ : $\quad$ order of the polynomial (1)

$M: \quad$ order of the polynomial $A(s)$

$P: \quad$ number of RHP (right half-plane) zeros of $A(s)$

$Z$ : number of singular frequencies on the interval $0 \leq \omega<+\infty$ for a fixed $k_{P}$.

A necessary stabilizability condition for (1) is

$$
Z \geq \frac{1}{2} E(N-M+2 P)+1
$$

Proof. Consider the function $F(s)$ as defined in (6). Recall that its imaginary part for $s=j \omega$ represents the generator of singular frequencies (5). Hence the Mikhailov plot of function $F(j \omega)$ intersects the real axis exactly at $Z$ singular frequencies $\left\{\omega_{i}^{\prime}\right\}$. If $p(s)$ is Hurwitz, then appliance of the principle of argument on a large semicircle on the left-hand side of the $s$-plane with center at $s=0$ yields

$$
\Delta \phi_{F}=(N-M+2 P) \pi / 2
$$

where $\Delta \phi_{F}$ represents the phase change of the function $F(j \omega)$ on the imaginary axis for $0 \leq \omega<+\infty$. According to Lemma 3, as $\omega$ changes within $0 \leq \omega<+\infty, F(j \omega)$ will intersect the real axis at least $\frac{1}{2} E(N-M+2 P)+1$ times.

Using Lemma 3 the theorem is directly extendable for the case when $A(s)$ possesses zeros on the imaginary axis. In the forthcoming theorems applies the notation introduced in Theorem 3.
Theorem 4: Suppose $A(s)$ has $L$ zeros on the imaginary axis. Then, for the polynomial (1) to be stable

(a) if $A(0) \neq 0$

$$
Z \geq \frac{1}{2} E(N-M+2 P+L)+1
$$

(b) if $A(0)=0$

$$
Z \geq \frac{1}{2} E(N-M+2 P+L)
$$

singular frequencies corresponding to a fixed $k_{P}$ are required within the interval $\omega \in[0,+\infty)$.

Notice that both, Theorem 3 and 4, include the singular frequency at $s=0$. Given that the existence of a singular frequency at $s=0$ can be implicitly read from the non-divergence of the plot of the generator of singular frequencies (5) at $\omega=0$, the next theorem simplifies the both results and disburdens them from the situation at $s=0$.

Theorem 5: Suppose $A(s)$ has $L$ zeros on the imaginary axis and consider the function $k_{P}=k_{P}(\omega)$ in (5). Fix $k_{P}=k_{P}^{*}$. Then for the polynomial (1) to be stabilizable, the function $k_{P}(\omega)-k_{P}^{*}$ must have

$$
Z \geq \frac{1}{2} E(N-M+2 P+L)
$$

positive zero frequencies.

\section{B. Time-delay systems}

The necessary stabilizability condition for the time-delay systems is derived based on the theorem on real-roots of quasi-polynomials, see [2]. The stabilizability theorem reads:

Theorem 6: Consider the quasipolynomial (7). Assume that $A(s)$ has no zeros on the imaginary axis, and $P$ RHP zeros. If (7) is Hurwitz-stabilizable then a $k_{P}$ and a $k \in \mathbb{N}$ exist, such that for $l \geq k$, the equation (9) possesses

$$
Z \geq \frac{1}{2} E(4 l+N-M+2 P)
$$

real roots in the interval $0<\omega<(2 l \pi+\delta) / T_{d}$, whereby $0<\delta<2 \pi$ is chosen such that the principal term of the quasi-polynomial (7) does not vanish at $\omega=( \pm 2 l \pi+\delta) / T_{d}$.

Its proof, as well as, the generalization for the case that $A(s)$ has zeros on the imaginary axis are similar to that in the Subsection III-A.

\section{Schur-stability}

Lemma 4: Consider the Mikhailov plot of a real-rational function $F(z)$ on the unity circle $\Gamma$ in (10) and let $L$ be the number of poles of $F(z)$ on $\Gamma$. If the phase change of the Mikhailov vector $F(z)$ over $\Gamma$ is $N \pi$, whereby the $L$ poles are left circumscribed, then it cuts the real axis at $Z$ frequencies $\alpha \in(0, \pi)$ 
(a) if $L$ is odd

$$
Z \geq \frac{N-L-1}{2}
$$

(b) otherwise

$$
Z \geq \frac{N-L-2}{2}
$$

Theorem 7: Consider the time-discrete polynomial (11) and let $E_{\Gamma}(z)$ be its decoupling function over the $\Gamma$ unity circle (10). Let

$N$ : order of the polynomial in (11)

$R: \quad$ number of zeros of $A(z) E_{\Gamma}(z)$ lying inside $\Gamma$

$L: \quad$ number of zeros of $A(z) E_{\Gamma}(z)$ lying on $\Gamma$

$Z: \quad$ number of real roots of (20) in the interval $0<\alpha<\pi$ for a fixed $r_{3}=r_{3}^{*}$.

If the polynomial (11) is Schur-stabilizable, then

(a) if $L$ is odd

$$
Z \geq N-R-\frac{L+1}{2}
$$

(b) otherwise

$$
Z \geq N-R-\frac{L+2}{2}
$$

The proof of the latter theorem is based on Lemma 4 and the principle of argument. It follows the same lines as the proof of Theorem 3.

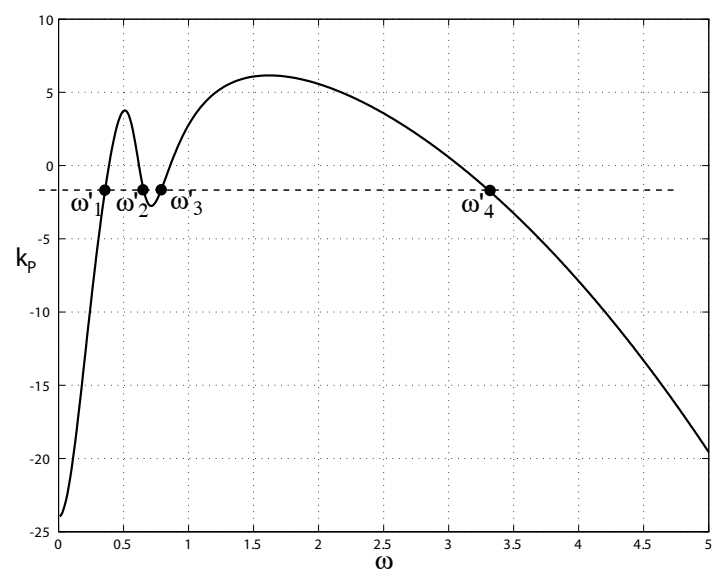

Fig. 1. Example 1; the generator of singular frequencies

\section{EXAMPLES}

Example 1. Consider the polynomial (1) with

$$
\begin{aligned}
& A(s)=-0.5 s^{4}-7 s^{3}-2 s+1 \\
& B(s)=s^{7}+11 s^{6}+46 s^{5}+95 s^{4}+109 s^{3}+74 s^{2}+24 s .
\end{aligned}
$$

Its generator of singular frequencies (5) reads

$$
k_{P}=\frac{-96+1028 \omega^{2}-2968 \omega^{4}+2534 \omega^{6}-216 \omega^{8}-2 \omega^{10}}{4-116 \omega^{4}+\omega^{8}+16 \omega^{2}+196 \omega^{6}}
$$

and is depicted in Fig. 1. According to the definitions in Theorem 3, $N=7, M=4$ and $P=1$. Theorem 5 claims that for stability, a $k_{P}$ must exist in Fig. 1, such that $Z \geq \frac{1}{2} E(N-M+2 P)=2$ real-roots in (35) exist within $0<\omega<+\infty$. By observation of the plot in Fig. 1 it is obvious that this condition is fulfilled for $-24<k_{P}<6.1565$. Indeed, the reader may check that stable PID controllers exist within this $k_{P}$-interval. E.g. such a stable PID controller is $k_{P}=-2, k_{I}=5, k_{D}=10$.

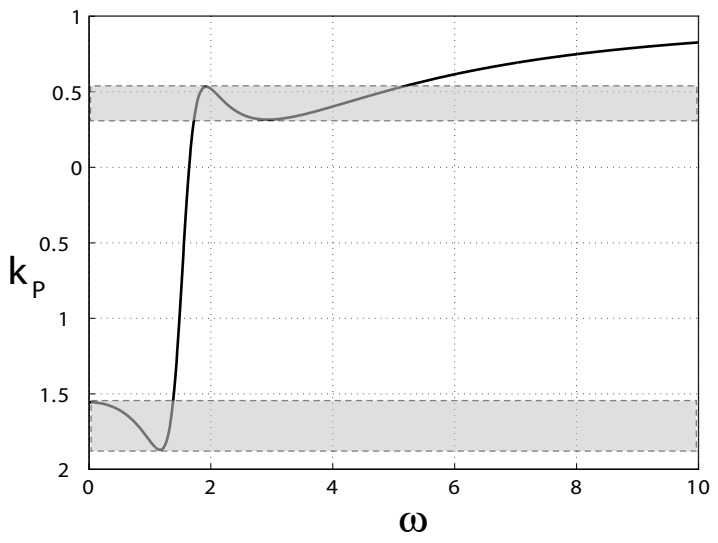

Fig. 2. Example 2; the generator of singular frequencies

Example 2: Shifted stable $k_{P}$-intervals. Let

$$
\begin{aligned}
& A(s)=s^{3}+3 s^{2}+9, \\
& B(s)=s^{4}+2 s^{4}+3 s^{2}+7 s+14 .
\end{aligned}
$$

In this case, $N=4, M=3$ and $P=2$, and for a fixed $k_{P}, Z \geq \frac{1}{2} E(N-M+2 P)=2$ real roots in (5) are required within $0<\omega<+\infty$. Now consider Fig. 2, where the plot of the generator of singular frequencies for $\omega \geq 0$ is depicted. The two (shaded) $k_{P}$-intervals of interest are directly recognized to fulfill the stabilization condition. For other $k_{P}$ 's no stable PID controllers exist; Fig. 3 shows the total set of PID stabilizers.

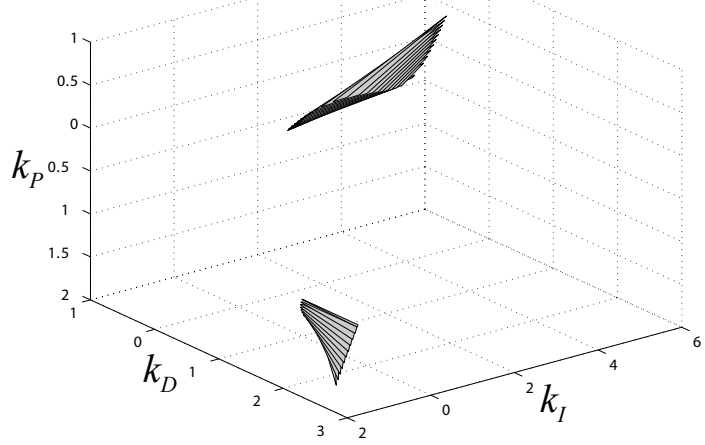

Fig. 3. Example 2; the set of all PID stabilizers 
Example 3: Missing stability. Let

$$
\begin{aligned}
& A(s)=1 \\
& B(s)=s^{5}+s^{4}-3 s^{3}-s^{2}+2 s .
\end{aligned}
$$

Theorem 3 requires at least 2 positive root frequencies, however for $-2>k_{P}$, just 1 exists, otherwise none. Thus, polynomial (1) is unstable no matter what $k_{P}, k_{I}, k_{D}$.

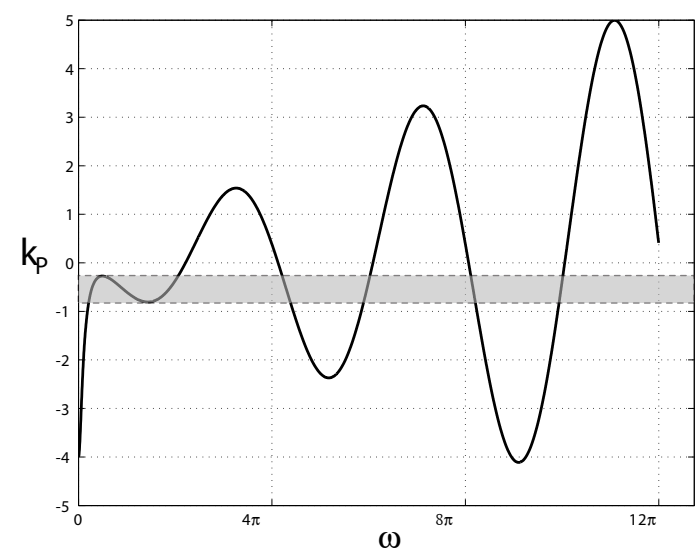

Fig. 4. Example 4; the generator of singular frequencies

Example 4: Time-delay system. Consider the quasipolynomial (7) with

$$
\begin{aligned}
& A(s)=-7 s^{2}-2 s+1 \\
& B(s)=s^{4}+3 s^{3}-3 s^{2}+4 s
\end{aligned}
$$

and $T_{d}=0.5$. In this case $N=4, M=2$, and $P=2$. According to Theorem 6 a necessary condition for stabilizability of (7) is to find a sufficiently large $k$ such that for a fixed $k_{P}$ in Fig. 4 within any interval $0<\omega<4 l \pi+\delta$, with $l \geq k$, and $0<\delta<4 \pi$, at least $E(2 l+3)=2 l+2$ real frequency roots are available. By observation of the plot in Fig 4 it is easily checked that already for $k=1$ and $\delta=\pi$, the condition is fulfilled within the shaded interval $-0.8<k_{P}<-0.27$. For all other values of $k_{P}$ no stable controllers exist.

Example 5: Schur-stability. The characteristic polynomial (11) is considered with

$$
\begin{aligned}
A= & z^{5}+9.44 z^{4}-5.34 z^{3}-9.34 z^{2}+5.04 z+\cdots \\
& 0.59 \\
B= & 0.19 z^{8}-0.73 z^{7}+z^{6}-0.45 z^{5}-0.12 z^{4}+\cdots \\
& 0.14 z^{3}-0.009 z^{2}-0.008 z .
\end{aligned}
$$

The polynomial $A(z)$ possesses three zeros inside the Schur-circle $\Gamma$, one zero at $z=-1$ and one zero outside $\Gamma$. For the decoupling function $E_{\Gamma}(z)=z$, see Theorem 1 , $N=8, \quad R=3+1=4$, and $L=1$. Hence, for stabilizability a $r_{3}$ must exist in (20) with $Z \geq N-R-\frac{L+1}{2}=3$ zero frequencies in $0 \leq \alpha \leq+\pi$. By observing the plot in Fig. 5, it can be discriminated that this condition is fulfilled within $-0.52236<r_{3}<0.00290$. Furthermore, zooming

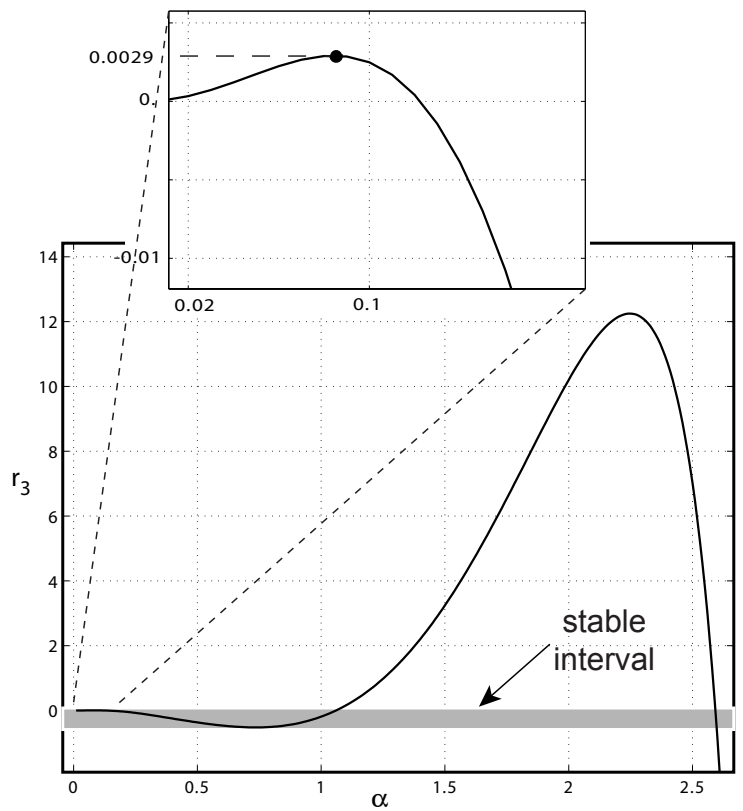

Fig. 5. Example 5; the generator of singular frequencies

of plot in Fig. 5 identifies for $0<r_{3}<0.00290$ two additional zero frequencies. Alternatively, if the decoupling function $E_{\Gamma}(z)=1+z^{2}$ is used then $N=8, \quad R=$ 3 , and $L=3$, i.e. again for stability $Z \geq N-R-\frac{L+1}{2}=3$ are required within $0 \leq \alpha \leq+\pi$.

\section{CONClusions}

This paper provides a necessary condition for Hurwitzstability of a PID loop. The condition is easily checked by observation of a plot $k_{P}=k_{P}(\omega)$. Thereby for a fixed $k_{P}$ a minimal number of real root-frequencies is required in $(0,+\infty)$. The condition is extended for discrete-time PID controllers and PID control loops with time-delay. The usability of the rule is illustrated by several examples.

\section{REFERENCES}

[1] BAJCINCA, N.: The method of singular frequencies for robust design in an affine parameter space, 9th Mediterranean Conference on Control and Automation, 2001 Dubrovnik.

[2] Pontryagin, L.S.: On the Zeros of Some Elementary Transcendental Functions, American Mathematical Society Translations, pp. 95-110.

[3] BajCinca, N., T. Hulin: RobSin: A new tool for robust design of PID and three-term controllers based on singular frequencies Proc. CACSD/CCA/ISIC, 2004 Taipei.

[4] Ackermann, J., D. Kaesbauer and N. Bajcinca: Discrete-time robust PID and Three-Term control, Proc. XV IFAC World Congress, 2002 Barcelona.

[5] Ackermann, J.; Kaesbauer, D. Design of robust PID controllers Proc. European Control Conference, Porto, 2001.

[6] Soylemez, M.T.; Munro, N; BAKI, H.: Fast calculation of stabilizing PID controllers. Automatica 39, S. 121-126.

[7] AströM, K.J., T. HÄGGLUnd: PID controllers: Theory, design and tuning, Instrument Society of America, Research Triangle Park, North Carolina, 2nd Edition, 1995.

[8] Ziegler, J.G., N.B. Nichols: Transactions of the ASME, November 1942. 